\title{
Analytical Solution of Transients in Single-Line-to-Ground Faults in Three-Phase Power Systems
}

\author{
Diego Bellan \\ Department of Electronics, Information and Bioengineering \\ Politecnico di Milano \\ Milan, Italy \\ diego.bellan@polimi.it
}

\begin{abstract}
This paper deals with the analytical evaluation of the transient current in single-line-to-ground fault in a threephase power system. The proposed methodology, in contrast with conventional numerical techniques, is based on the Clarke transformation working in the time domain. Due to the system asymmetry introduced by the single line fault, the modal circuits alpha, beta, and zero, related to the Clarke transformation, result in coupled circuits. A simple circuit representation of such coupling is derived, allowing straightforward evaluation of the fault current in the Laplace domain. Time domain behavior obtained through inverse Laplace transform is validated by means of Simulink time-domain simulation of single line faults.
\end{abstract}

Keywords-Clarke transformation, power system analysis, time-domain analysis, transient analysis

\section{INTRODUCTION}

Analytical calculation of asymmetrical fault transients in three-phase power systems is a critical issue in power system analysis because the conventional circuit techniques leading to decoupled modal circuits (i.e., symmetrical components and Clarke transformations) assume system symmetry as basic hypothesis [1]-[5]. A single-line-to-ground fault introduces a system asymmetry resulting in coupled modal circuits when the above mentioned transformations are used [6]-[8]. For this reason analysis of asymmetrical faults is commonly performed by means of numerical tools such as the well-known commercial simulator ElectroMagnetic Transients Program (EMTP) [9]. A numerical approach can tackle the problem by solving the circuit through general circuit techniques (i.e., techniques not specific to three-phase circuits) [9]-[11] or by introducing some approximate equivalent circuits [12]-[14]. In both cases, a numerical approach prevents a deeper theoretical/physical insight into the system under analysis, and the obtained solutions are affected by numerical/modeling approximations.

In this paper, in contrast with the numerical methods mentioned above, an analytical and rigorous approach is introduced for the evaluation of the transient current in a single-line-to-ground fault. The proposed theoretical methodology is based on the Clarke transformation in the rational form. It is shown that the asymmetry introduced by the fault can be represented by an equivalent circuit consisting in a simple ideal transformer coupling the alpha and zero modal circuits. The Laplace transform can be conveniently used to represent the circuit equivalents at the fault location. Basic circuit techniques and the inverse Laplace transform allow the straightforward evaluation of the fault current in the time domain.

The paper is organized as follows. In Section II the rational Clarke transform is briefly recalled. In Section III the proposed methodology and the related equivalent circuits are derived. In Section IV the proposed theoretical approach is validated by means of Simulink time-domain simulations of a single-line-to-ground fault. Concluding remarks are discussed in Section V.

\section{BACKGROUND: THE ClARKE TRANSFORMATION}

The Clarke transformation operates on three phase variables (i.e., currents or voltages) in the time domain to obtain the transformed variables alpha, beta, and zero, according to:

$$
\left[\begin{array}{l}
i_{\alpha} \\
i_{\beta} \\
i_{0}
\end{array}\right]=\sqrt{\frac{2}{3}}\left[\begin{array}{ccc}
1 & -1 / 2 & -1 / 2 \\
0 & \sqrt{3} / 2 & -\sqrt{3} / 2 \\
1 / \sqrt{2} & 1 / \sqrt{2} & 1 / \sqrt{2}
\end{array}\right]\left[\begin{array}{l}
i_{a} \\
i_{b} \\
i_{c}
\end{array}\right]
$$

Notice that (1) is the so-called rational form of the Clarke transformation since the orthogonality of the transformation matrix results in the conservation of power from the $(a, b, c)$ to the $(\alpha, \beta, 0)$ variables. This property is fundamental in the derivation of equivalent circuits in the transformed domain. Moreover, notice that (1) holds also when linear operators are applied to the time domain variables, e.g., phasors under sinusoidal steady state and Laplace transform for general time-domain behavior.

In view of the transient analysis described in Section III, it is important to stress that the phasor analysis under sinusoidal conditions is needed to solve the three-phase system in the steady state prior the fault in order to evaluate the initial conditions for the fault event. In fact, before the fault event, the system is assumed symmetrical and under sinusoidal steady state. Therefore, from the standard Clarke analysis, we obtain three uncoupled circuits whose phasor solutions can be readily derived. Such solutions allow the calculation of the initial conditions corresponding to the fault time instant. The initial conditions will be used for a proper application of the Laplace transform to analyze the transient after the fault event.

\section{CIRCUIT REPRESENTATION OF A SINGLE-LINE-TO- GROUND FAULT}

Let us consider the reference three-phase circuit shown in Fig. 1 where the left side represents a simplified model for the source and the line, whereas the right side represents the load. The single-line-to-ground fault is taken into account by a three-phase switch where only the switch between line $a$ and ground can be operated, whereas the remaining switches $b$ and $\mathrm{c}$ hold the open-circuit state. Prior the fault event, the circuit is working under sinusoidal steady state conditions. Phasors can be used to evaluate the currents in the alpha, beta, and zero circuits obtained through the Clarke transformation. From the 


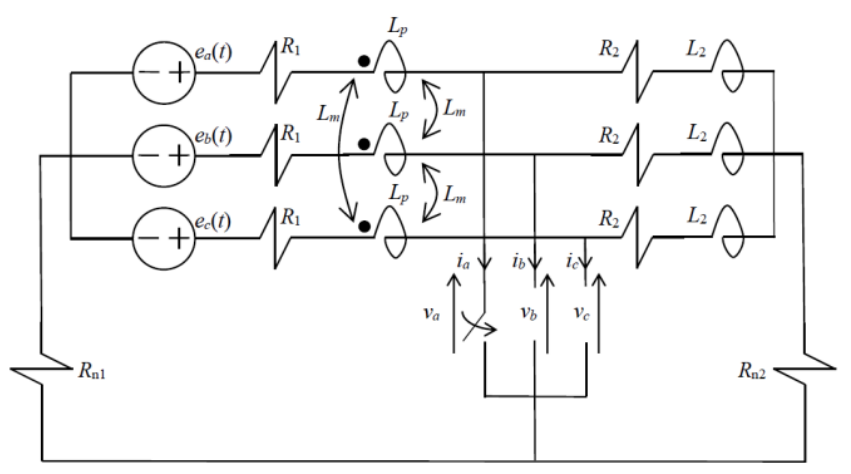

Fig. 1. Reference three-phase circuit used to explain the proposed methodology.
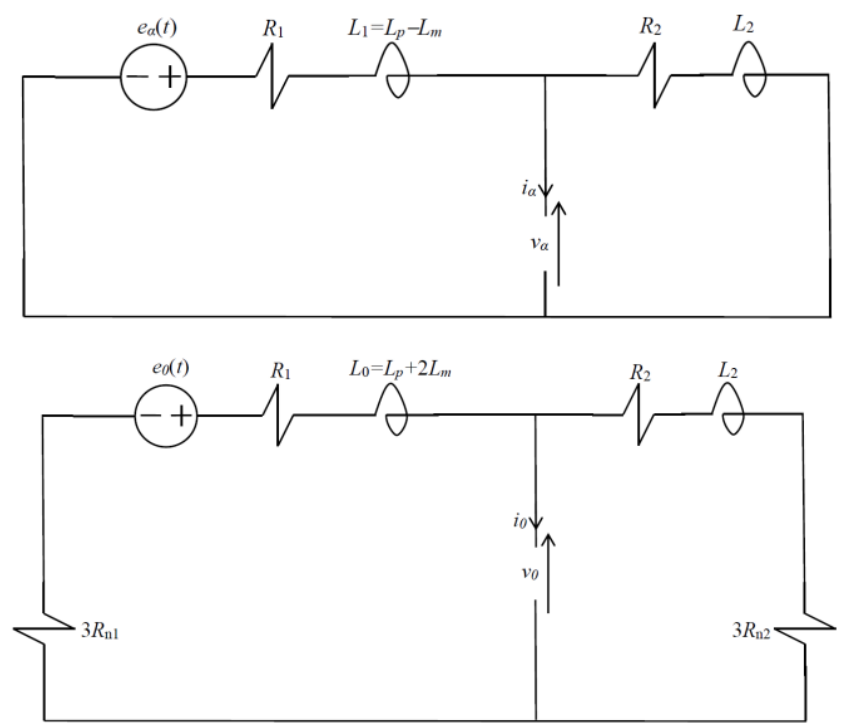

Fig. 2. Alpha and zero circuits for $t<0$, i.e., prior the fault event. They can be analyzed in the phasor domain in order to calculate the inductors currents at $t$ $=0$, i.e., the initial conditions for the subsequent transient analysis.

phasor solutions the line currents at $t=0$ (i.e., the fault time instant) can be readily evaluated in order to establish the initial conditions for the inductors. The alpha and zero circuits in the sinusoidal steady state prior the fault event are shown in Fig. 2. The beta circuit is not shown because it has the same topology as the alpha circuit.

Once the initial conditions for the alpha, beta, and zero circuits have been evaluated, the fault takes place, i.e., the switch $a$ in Fig. 1 is operated. The constraints set by the threephase switch on the $a, b, c$ variables:

$$
v_{a}=0, \quad i_{b}=i_{c}=0
$$

result in constraints on the alpha, beta, zero variables according to the Clarke transformation:

$$
\begin{aligned}
{\left[\begin{array}{l}
v_{\alpha} \\
v_{\beta} \\
v_{0}
\end{array}\right]=} & \sqrt{\frac{2}{3}}\left[\begin{array}{ccc}
1 & -1 / 2 & -1 / 2 \\
0 & \sqrt{3} / 2 & -\sqrt{3} / 2 \\
1 / \sqrt{2} & 1 / \sqrt{2} & 1 / \sqrt{2}
\end{array}\right]\left[\begin{array}{c}
0 \\
v_{b} \\
v_{c}
\end{array}\right]= \\
= & \sqrt{\frac{2}{3}}\left[\begin{array}{l}
-1 / 2\left(v_{b}+v_{c}\right) \\
\sqrt{3} / 2\left(v_{b}-v_{c}\right) \\
1 / \sqrt{2}\left(v_{b}+v_{c}\right)
\end{array}\right]
\end{aligned}
$$

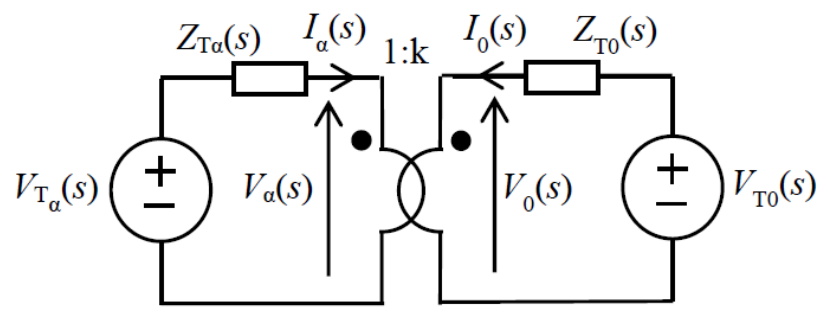

Fig. 3. Coupled alpha and zero circuits in the Laplace domain for fault current analysis.

$$
\begin{aligned}
& {\left[\begin{array}{l}
i_{\alpha} \\
i_{\beta} \\
i_{0}
\end{array}\right]=\sqrt{\frac{2}{3}}\left[\begin{array}{ccc}
1 & -1 / 2 & -1 / 2 \\
0 & \sqrt{3} / 2 & -\sqrt{3} / 2 \\
1 / \sqrt{2} & 1 / \sqrt{2} & 1 / \sqrt{2}
\end{array}\right]\left[\begin{array}{c}
i_{a} \\
0 \\
0
\end{array}\right]=} \\
& =\sqrt{\frac{2}{3}}\left[\begin{array}{c}
i_{a} \\
0 \\
i_{a} / \sqrt{2}
\end{array}\right]
\end{aligned}
$$

Thus, from (3a)-(3b) we obtain the following constraints on the Clarke variables:

$$
v_{0}=-\sqrt{2} v_{\alpha}, \quad i_{\alpha}=\sqrt{2} i_{0}, \quad i_{\beta}=0
$$

The first two equations in (4) define an ideal transformer with ratio $k=-\sqrt{2}$ between alpha and zero circuits, whereas the third equation states that the beta circuit is not involved in the transient. Therefore, the transient analysis can be performed in the Laplace domain by representing the coupling between alpha and zero circuits through an ideal transformer. The equivalent circuit is represented in Fig. 3, where the Thevenin equivalents of the alpha and zero circuits shown in Fig. 2 are considered. Simple circuit analysis of Fig. 3 provides:

$$
I_{\alpha}(s)=\frac{V_{T \alpha}(s)-V_{T 0}(s) / k}{Z_{T \alpha}(s)+Z_{T 0}(s) / k^{2}}=\frac{V_{T \alpha}(s)+V_{T 0}(s) / \sqrt{2}}{Z_{T \alpha}(s)+Z_{T 0}(s) / 2}
$$

Finally, from ( $3 b)$ we obtain the Laplace transform of the fault current $I_{a}(s)=\sqrt{3 / 2} I_{\alpha}(s)$.

\section{NUMERICAL VALIDATION}

The three-phase circuit shown in Fig. 1 was implemented in Simulink and simulated in the time domain. The switch $a$ was operated at $t=0$ and the transient of the fault current $i_{a}(t)$ was calculated. The source was a set of balanced voltages with positive phase rotation, phase of $e_{a}(t)$ equal to $\pi / 6$, amplitude $1 \mathrm{kV}(\mathrm{rms})$ and frequency $50 \mathrm{~Hz}$. The other parameters were selected as $R_{1}=R_{2}=R_{n 1}=R_{n 2}=0.1 \Omega, L_{p}=3 \mathrm{mH}$, $L_{m}=1 \mathrm{mH}, L_{2}=5 \mathrm{mH}$.

Fig. 4a shows the comparison between $i_{a}(t)$ obtained by simulation (red curve) and obtained by inverse Laplace transform of $I_{a}(s)$ as calculated in Section III (blue curve). Notice that the red curve is exactly covered by the blue curve. Thus, the proposed analytical approach is validated. The black curve was obtained by using a well-known approximate approach consisting in the evaluation of the transient of the positive sequence (PS) circuit only (i.e., the PS model) [12], [14]. Notice that the PS provides an underestimate of the actual fault current.

In Fig. $4 \mathrm{~b}$ the same comparison was performed by assuming the phase of source $a$ equal to $-\pi / 2$ instead of $\pi / 6$ as in the previous case. Also in this case the proposed analytical 


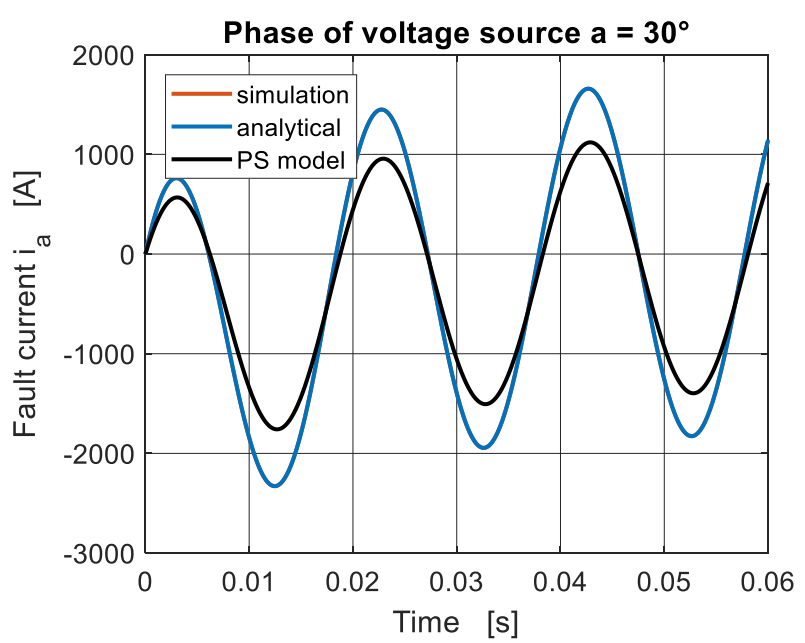

(a)

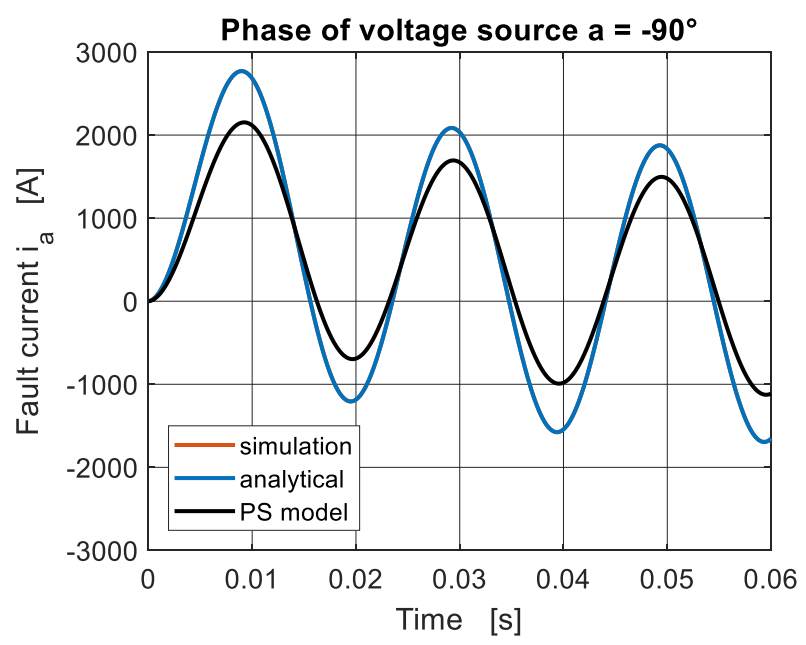

(b)

Fig. 4. Comparison between numerical simulation (red curves), analytical model (blue curves), and positive sequence model (black curves) for the transient behavior of the fault current $i_{a}(t)$ in the three-phase circuit in Fig. 1. In (a) the phase of the voltage source $e_{a}(t)$ was $\pi / 6$, whereas in (b) the phase was $-\pi / 2$. Red curves are covered by blue curves.

approach shows agreement with Simulink numerical results. The approximate PS model provides significant underestimates also in this case.

\section{CONCLUSION}

An analytical method based on the solution of coupled Clarke circuits in the Laplace domain was proposed for the evaluation of the transient current in single-line-to-ground faults in three-phase circuits. Despite the strong asymmetry introduced by the fault, the circuit coupling can be managed quite easily since it was shown that it consists in an ideal transformer connected across the alpha and zero circuits. It is worth noticing that such result is a consequence of the Clarke transformation in its rational form. The proposed analytical approach provides a consistent methodology for analytical solution of time-domain transients and allows theoretical insight into the phenomenon, in contrast with the commonly adopted numerical approach.

Thus, the main contribution of this paper can be regarded as a theoretical and methodological contribution, whereas the accuracy of the transient evaluation is not affected with respect to existing numerical techniques. Future work will be devoted to investigate other kinds of asymmetrical faults and more extensive numerical validation.

\section{REFERENCES}

[1] G. C. Paap, "Symmetrical components in the time domain and their application to power network calculations," IEEE Trans. on Power Systems, vol. 15, pp. 522-528, 2000.

[2] J. Aller, A. Bueno, and T. Paga, "Power system analysis using space vector transformation," IEEE Transactions on Power Systems, vol. 17, pp. 957-965, 2002.

[3] G. Superti-Furga, S. Barcellona, and E. Tironi, "Space-vector approach in three-phase unbalance and distortion analysis," Proc. of 17 th International Conference on Harmonics and Quality of Power (ICHQP), pp. 721-726, Belo Horizonte, Brazil, 2016.

[4] D. Bellan, S. A. Pignari, and G. Superti-Furga, "Consistent circuit technique for zero-sequence currents evaluation in interconnected single/three-phase power networks," Journal of Electrical Systems, vol. 12, pp. 230-238, 2016.

[5] D. Bellan and G. Superti-Furga, "Space-vector state-equation analysis of three-phase transients," Journal of Electrical Systems, vol. 14, pp. 188-198, 2018.

[6] M. Owen, "Transient analysis using component transforms," Proc. of 2011 IEEE Power Engineering and Automation Conference, pp. 4-9, 2011.

[7] D. Bellan, G. Superti-Furga, and S. A. Pignari, "Circuit representation of load and line asymmetries in three-phase power systems," International Journal of Circuits, Systems and Signal Processing, vol. 9, pp. 75-80, 2015.

[8] D. Bellan, "Transient analysis of single-line-to-ground faults in threephase circuits using Clarke transformation," in Proc. of International Conference on Green Energy for Sustainable Development (ICUE 2018), pp. 1-4, Phuket, Thailand, 2018.

[9] J. Mahseredjian, V. Dinavahi, and J. A. Martinez, "Simulation tools for electromagnetic transients in power systems: overview and challenges," IEEE Trans. on Power Delivery, vol. 24, pp. 1657-1669, 2009.

[10] C. Dufour, J. Mahseredjian, and J. Belanger, “A combined state-space nodal method for the simulation of power system transients," IEEE Trans. on Power Delivery, vol. 26, pp. 928-935, 2011.

[11] F. Plumier, P. Aristidou, C. Geuzaine, and T. Van Cutsem, "Cosimulation of electromagnetic transients and phasor models: a relaxation approach," IEEE Trans. on Power Delivery, vol. 31, pp. 2360-2369, 2016.

[12] W. P. Lewis, "Solution of network transients using symmetricalcomponent techniques," Proc. IEE, vol. 113, pp. 2012-2016, 1966.

[13] A. Greenwood, Electrical transients in power systems, John Wiley \& Sons, Inc., 1991.

[14] J. Peralta, F. de Leon, and J. Mahseredjian, “Assessment of errors introduced by common assumptions made in power system studies," in Proc. of IEEE Power and Energy Scociety General Meeting, pp. 1-8, San Diego, CA, USA, 2011. 